\title{
Foot and Mouth Disease
}

\author{
Elsayed Ebrahime Elsayed Elmeligy* \\ Faculty of Veterinary Medicine, Kafr ElSheikh University, Egypt
}

\begin{abstract}
Received: 01 August, 2017; Accepted:03 August, 2017; Published: 04 August, 2017
${ }^{*}$ Corresponding author: Elsayed Ebrahime Elsayed Elmeligy, Faculty of Veterinary Medicine, Kafr ElSheikh University, Egypt; E-mail: elsayedebrahime@gmail.com
\end{abstract}

\section{Overview}

Foot and Mouth Disease (FMD) is a highly communicable viral disease caused by an Aphthovirus of the family Picornaviridae [1]. There are seven strains (A, O, C, SAT1, SAT2, SAT3, Asia1) each one requiring a specific vaccine strain to provide immunity to a vaccinated animal, Six of the 7 serotypes have occurred in Africa (O, A, C, SAT-1, SAT-2, SAT-3), 4 in Asia (O, A, C, Asia-1), and 3 in South America (O, A, C). FMD disease is a severe, highly contagious viral disease of cattle and swine. It also affects sheep, goats, deer, and other cloven-hooved ruminants, all species of deer and antelope as well as elephant, and giraffe are susceptible to FMD.

FMD is endemic in several parts of Asia, most of Africa and the Middle East. In Latin America, the majority of countries applied zoning and are recognized free of FMD with or without vaccination, and the disease remains endemic in only a few countries. Australia New Zealand and Indonesia, Central and North America and continental Western Europe are currently free of FMD. However, FMD can occur sporadically in typically free areas [2] (Figure 1).

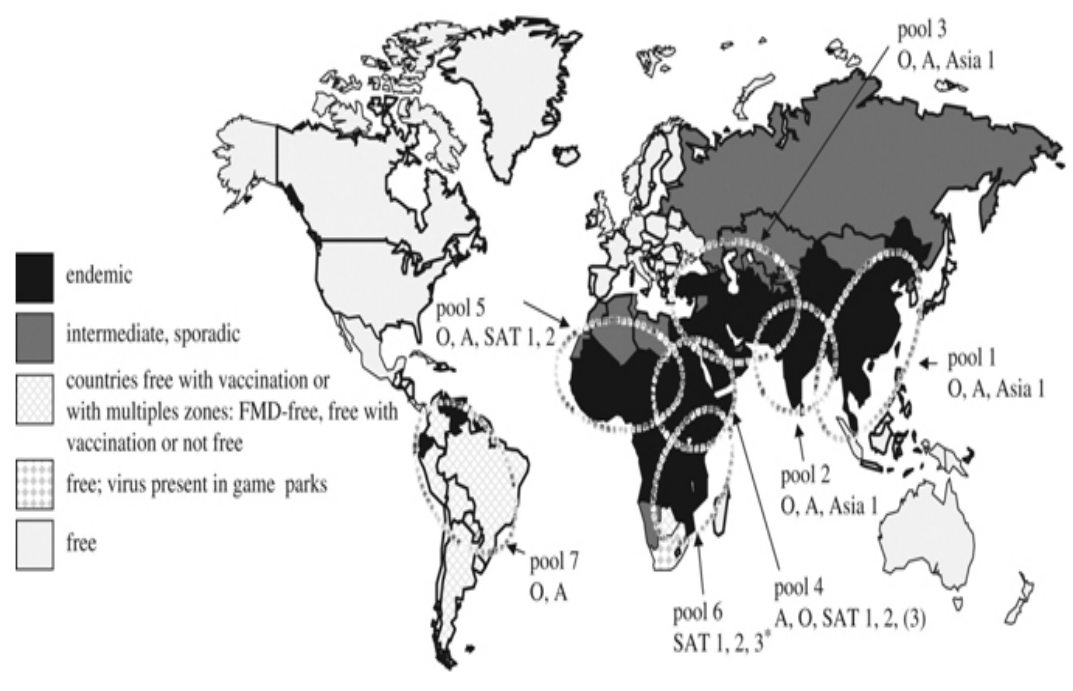

Figure 1: Conjectured national FMD status in 2008 overlaid with regional FMDV pools and predominant virus serotypes

\section{Transmission}

FMD is found in all excretions and secretions from an infected animal. The virus may be present in milk and semen for up to 4 days before the animal shows clinical signs of disease. Transmission of FMDV occurs by direct contact between infected and susceptible animals [3].

$$
\text { Virus spread through any or all of the following }
$$

-New animals carrying the virus (saliva, milk, semen, etc.) may introduce the disease to a herd.

-Contaminated pens, buildings or vehicles used to house and move susceptible animals. -Contaminated materials such as hay, feed, water, milk or biologics.

-People wearing contaminated clothes or footwear, or using contaminated equipment.

-Meat or animal products, raw or improperly cooked food infected with the virus and fed to susceptible animals.
-Aerosol spread of virus from an infected property via air currents.

- Humans normally do not acquire FMD, but the serotypes that have been isolated include $\mathrm{O}$ (most commonly), C and rarely A. Direct contact may result in infection, as well as ingestion of unprocessed milk or dairy products from infected animals (extremely rare) For that reasons, FMD is not considered to be a zoonotic disease.

\section{Incubation period}

FMD has one of the shortest incubation periods of any major infectious disease known. The incubation period for clinical signs is usually 3-5 days. However, once an active outbreak of FMD occurs and there is a large amount of FMDV in the environment, incubation periods of as short as 24-36 hours are seen. Experimentally, clinical signs can be seen as early as 12 hours post exposure [4].

\section{Morbidity}

Morbidity is usually very high (close to $100 \%$ ) in fully susceptible cloven-hoofed domestic animals and quite variable from high to very low in wildlife animals depending on the FMDV sub-type and the species involved.

\section{Mortality}

Mortality due to FMDV infections in general is very low in adult animals (1-5 \%) but higher in young calves, lambs and piglets (20\% or higher).

\section{Clinical Signs}

-Fever; FMDV infected cattle will develop fever of $103^{\circ}-105^{\circ} \mathrm{F}\left(39.4^{\circ}-40.6^{\circ} \mathrm{C}\right)$ accompanied by dullness, but in swine $104^{\circ}-105^{\circ} \mathrm{F}\left(40.0^{\circ}-40.6^{\circ} \mathrm{C}\right)$.

-Drop in milk production.

-Loss of appetite and weight loss.

-Excessive drooling of saliva, the saliva may look thicker with long strands, and more foaming due to the dehydration of the febrile animal and presence of excess nasal mucous secretions (figure 2).

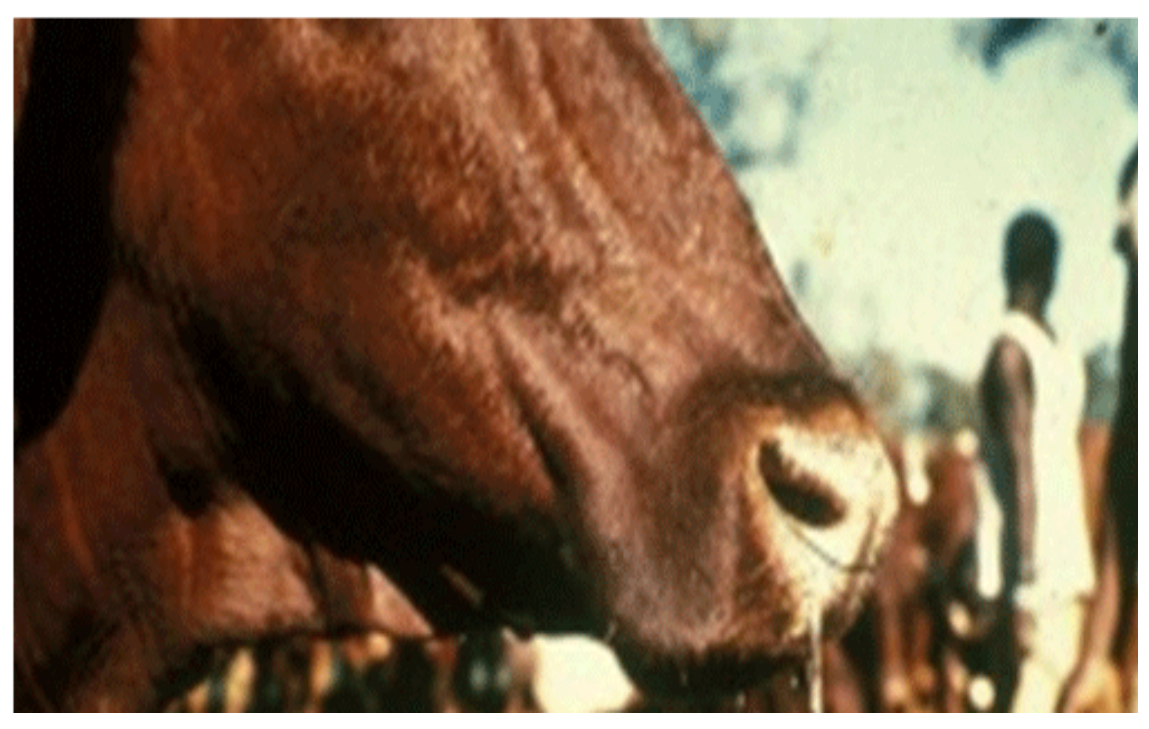

Figure 2: In cattle a mucoid nasal discharge develops 
-Due to inability of the animals to swallow the saliva or clean the nostrils with the tongue as there is a painful mouth lesions.

-Vesicles are formed in the nacres, lips, gums, tongue, teats, and interdigital spaces (figure $3)$.

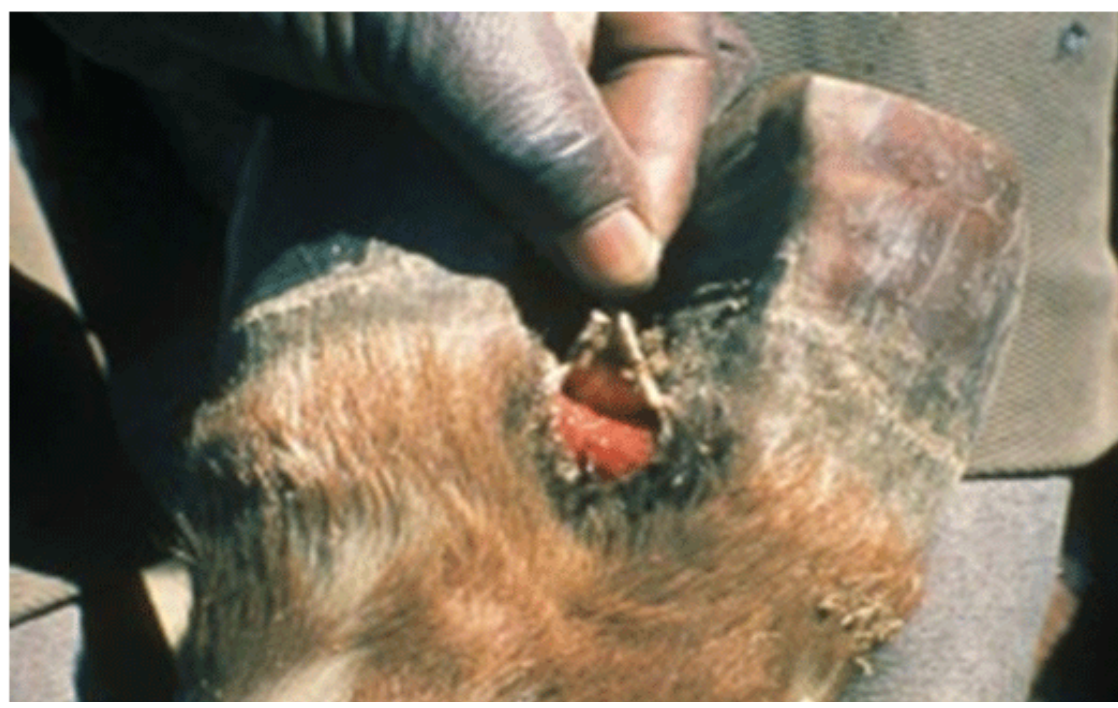

Figure 3: Lesions develop in the skin of the interdigital space

-When vesicles rupture, erosions form in the same locations in the mouth and hard palate when this lesions progress there may be more marked drooling and lameness.

-Pregnant cows may abort and young calves may die without developing any vesicular lesions (figure 4)

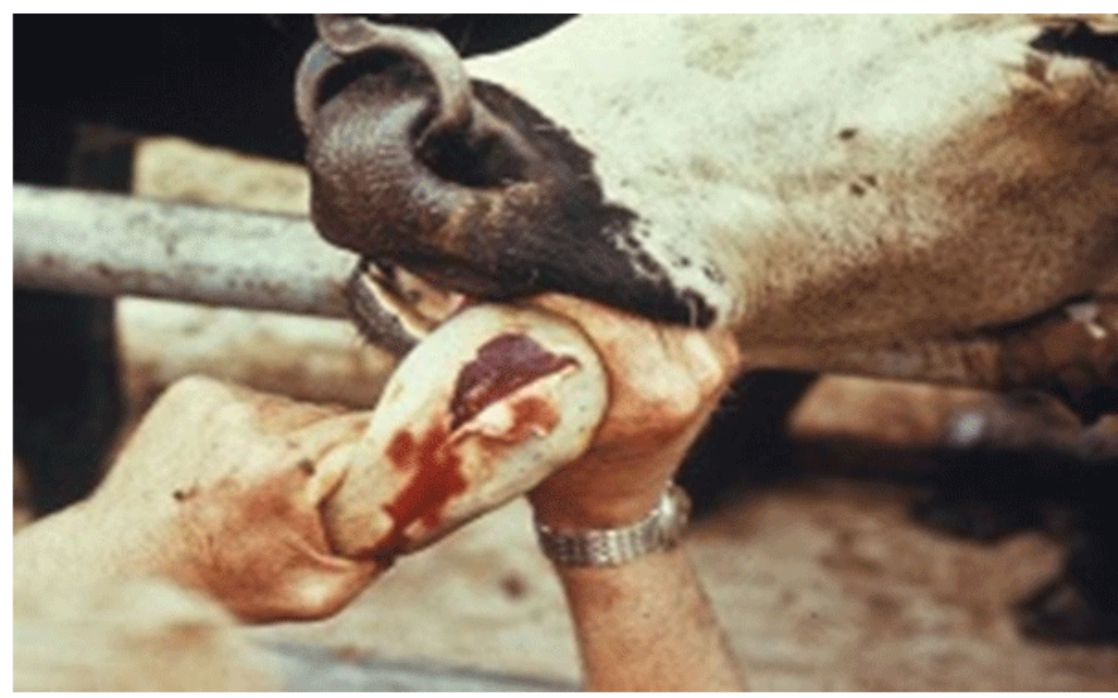

Figure 4: Epithelial separation can leave lesions on the tongue up to $60 \mathrm{~mm}$ in diameter

\section{Lab Diagnosis}

The ideal samples for FMD (or any other vesicular disease condition) are: vesicular fluid, vesicular epithelium, scrapings or deep swabs of erosions, nasal swabs and acute and convalescent serum samples. Esophagealpharyngeal fluid collected by means of a probang cup is used for the detection of FMDV carrier animals. Virus isolation and Enzyme-Linked Immunosorbent Assay (ELISA) are the gold standard tests for diagnosis of FMD for the diagnosis of FMD or other vesicular diseases include tests for the detection of virus (virus isolation), detection of viral antigens (Antigen-capture ELISA, Complement Fixation), detection of antibodies (virus neutralization, agar-gel immunodiffusion, antibody detection ELISA), or the new technology of nucleic acid detection (real time reverse transcriptase polymerase chain reaction - RRT-PCR) [5].

\section{Differential Diagnosis}

FMD in cattle is clinically identical to Vesicular Stomatitis (VS) requires that any bovine with lesions compatible to vesicular diseases must be tested for VS and FMD.

FMD in cattle also has some common features with a number of other diseases including: rinderpest, mucosal disease, bovine viral diarrhea, infectious bovine rhinotracheitis, bluetongue, bovine mammillitis, bovine papular stomatitis, and other chemical or traumatic diseases of the oral cavity and feet.

There is a condition in Uruguay and southern Brazil known as Bocopa (from Spanish boca $=$ mouth; cola $=$ tail; pata $=$ foot ) that has some similarities to FMD due to massive erosions on the mouth and feet. Bocopa is caused by an alkaloid toxin of the fungus Ramaria flavo-brunnescens that is seasonally present (February to June).

\section{Treatment, control and prevention}

Alough There is no specific treatment for FMD, but supportive care may be allowed for vesicles and erosions in mouth, tongue, legs and teats must be treated to prevent the secondary infection, the loose of hooves and enhance the ability to recover, the treatment should be applied locally and generally [6].

Localized treatment: rinse the ulcerated vesicles found on tongue, mouth, leg, hoof, teat with one of the following solution:

normal saline, acid citric $1 \%$ or potassium permanganate $1 \%$, alum $2 \%$, blue methylene $1 \%$ (or use juice of lemon for washing ulceration in mouth and tongue twice a day) after cleaning the wound in teats and limb with normal saline and drying apply antiseptic then ointment over the wound, Bandage all legs for the prevention of flies.

Generalized treatment: enhance immune system for increasing resistance to disease and stress condition to help up to recovery using antibiotic course treatment for secondary infections.

\section{Prevention and Control}

Prevention interventions include education and training on the recognition and diagnosis of FMD

Protection of FMD free countries, areas or zones is enhanced with stringent import and cross-border animal movement controls and surveillance [7].

-Maintain biosecurity practices to prevent introduction/spread of the virus.

-Measures that are recommended at the farm level include:

- Control over access to livestock by people and equipment.

- Control the introduction of new animals to existing stock.

-Maintain sanitation of livestock pens, buildings, vehicles and equipment.

-Monitor and report illness.

-Appropriate disposal of manure and dead carcasses.

\section{References}

1. David A. Ashford. Overview of Foot-and-Mouth Disease. Merck Manual Veterinary Manual.

2. List of FMD free Member Countries. OIE.

3. Arzt J, Juleff N, Zhang Z, Rodriguez LL. The Pathogenesis of Foot-and-Mouth Disease I: Viral Pathways in Cattle.Transbound Emerg Dis. 2011;58(4):291-304. doi: 10.1111/j.1865-1682.2011.01204.x

4. Foreign animal diseases, 7th ed, United States. Department of Agriculture, Animal and Plant Health Inspection

Service. 2008

5. Diagnosis of foot and mouth disease. Merck Veterinary Manual.

6. Variation among strains of foot and mouth disease. CAMPRIDGE University Press OIE/ FAO Global Conference on foot and mouth Disease -Final Recommendations.

7. OIE/FAO Global Conference on foot and mouth Disease -Final Recommendations 